\title{
A Cross-sectional Study on Hepatitis B Immune Status Among Vaccinated Healthcare Workers in Khartoum, Sudan
}

\section{Abdullahi Elsheikh Mahgoub ${ }^{1 *}$, Yassir Hamadalnil², Osama Mohammed ${ }^{3}$, Rasha Nakhleh",5, and Musa Mohamed Khair ${ }^{6}$}

1. Geriatric Fellow, Hurley Medical Center, Flint, Michigan, USA

2. Department of Clinical Microbiology, Faculty of Medicine, Nile University, Khartoum, Sudan

3. Central laboratory, Khartoum, Sudan

4. Geriatric Fellowship Program/Hurley Medical Center

5. College of Human Medicine, Michigan State University

6. Medicine, University of Khartoum, Khartoum, Sudan

Corresponding Author:

abdullahimahgoub@hotmail.com.

Received 18 January 2020

Accepted 07 March 2020

Published 31 March 2020

Production and Hosting by

Knowledge E

@)Abdullahi Elsheikh

Mahgoub. This article is

distributed under the terms

of the Creative Commons

Attribution License, which

permits unrestricted use and redistribution provided that

the original author and source are credited.

Editor-in-Chief

Prof. Mohammad A. M. Ibnouf

\section{Abstract}

Background: Hepatitis B virus (HBV) infection can lead to chronic hepatitis, liver cirrhosis, and Hepatocellular Carcinoma (HCC). Vaccination against the virus plays an important role in its prevention. Health personnel at risk of infection are recommended to receive the vaccine and to check their immune status thereafter.

Objective: To determine Hepatitis B Immune status among vaccinated health personnel in Khartoum state, Sudan. A total of 106 health personnel from different hospitals and doctors' mess in Khartoum and from different jobs were surveyed and their HBV surface antibodies levels were determined by HBsAb (Quantitative) ELISA Kits.

Methods: Of the 106 participants in this study, the majority were females $72.6 \%$. The mean age of those involved was 27.69 years, and nurses made for more than $50 \%$ of those surveyed. About $56.6 \%(60)$ of the health personnel involved took three or more doses of HBV vaccine while the rest received one or two doses. The results of the participants who received three or more doses of the vaccine represented immunity by $70 \%$. In general, about $47.2 \%$ (50) of the participants in this study did show excellent immune response $(>100 \mathrm{mlU} / \mathrm{mL})$ and 18.9\% (20) had weak protective levels (between 10 and $100 \mathrm{mlU} / \mathrm{mL}$ ) So, we concluded that HBV post-vaccination immunity status of health personnel in Khartoum state is lower than post-vaccination HBV immunity in other parts of the world. There is an urgent need for a national protocol of HBV vaccination to improve the immunization status of health personnel with a regular checkup of HBV immunity and easy access to a good-quality vaccine.

Key words: Hepatitis B vaccines; healthcare workers; immune response 


\section{Introduction}

Hepatitis B Virus (HBV) is a member of the Hepadnaviridae family with a small DNA virus and unusual features of replication similar to retroviruses, in which HBV can replicate through an RNA intermediate and forming a stable minichromosome (cccDNA) in the nucleus. These features of the HBV replication cycle give it the ability to persist in infected cells [1]. HBV can cause acute hepatitis and can also lead to other more serious complications like Chronic Hepatitis, Liver Cirrhosis, and Hepatocellular Carcinoma (HCC) [2]. HBV can survive outside the body for up to seven days; the virus incubation period is around two and a half months. The virus can be transmitted perinatally from a mother to her baby, horizontally by exposure to infected blood or blood products, or through direct spread percutaneously or to mucosal membranes [3]. HBV infects more than 300 million people worldwide; Sudan is classified among the countries with high HBV seroprevalence. Exposure to the virus varied from 47 to $78 \%$, with a hepatitis $B$ surface antigen prevalence ranging from $6.8 \%$ in central Sudan to $26 \%$ in southern Sudan [4]. HBV vaccine is a recombinant DNA vaccine that contains HBsAg genetically engineered from the yeast Saccharomyces cerevisiae. It provides a seroprotection rate of $85-100 \%$ that was seen one month after the last dose of vaccine and it confers immunity for at least 10 years [5]. Hepatitis $B$ vaccine is recommended for unvaccinated adults who are at risk for HBV infection like people whose sex partners have hepatitis B, sexually active persons who are not in a long-term monogamous relationship, persons seeking evaluation or treatment for a sexually transmitted disease, men who have sex with men, people who share needles, syringes, or other drug-injection equipment, household contacts of HBV-infected persons, health personnel and public safety workers at risk for exposure to blood or body fluids, residents and staff of facilities for developmentally disabled persons, persons in correctional facilities, victims of sexual assault or abuse, travelers to regions with increased rates of hepatitis $B$, people with chronic liver disease, patients on hemodialysis, HIV infection, or diabetes, and anyone who wants to be protected from HBV. The vaccine is usually given as three shots over a period of a six-month period [6]. Serum antibody to the hepatitis B surface antigen (anti-HBs) has long been established as a marker of vaccine-induced protection against hepatitis B. An anti-HBs level of $\geq 10 \mathrm{lU} / \mathrm{ml}$ has been suggested to indicate protection against hepatitis $B$ disease [7]. Old age in adults, male gender, increased body mass index, smoking, and concomitant disease are some of the factors that may decrease the immunologic response to HBV vaccine [8]. This study was conducted to determine hepatitis B immune status among vaccinated health personnel in Khartoum, Sudan. 


\section{Materials and Methods}

This is a descriptive cross-sectional study conducted at public hospitals of Khartoum including the Ibn Sina Specialized Hospital, the Alshaab Teaching Hospital, and the Academic Hospital from 30th July to 30th September 2017. The study included healthcare providers working in all departments, including trained nurses, medical attendants, and clinicians - both surgical and medical-related specialties and laboratory technicians. The investigator approached healthcare workers through the hospital administration and the heads of the departments. A self-administered questionnaire that covered age, gender, job description, total doses of vaccine and the date of the last dose plus other data such as duration since the last dose and a history of immunosuppressive treatment and blood transfusion was used to obtain information from the participants. The questionnaires were collected from the healthcare workers upon completion by appointment with the participant; $5 \mathrm{ml}$ venous blood sample was collected from the cubital vein under complete possible aseptic conditions in EDTA containers, centrifuged at 3000 rounds per min for $10 \mathrm{~min}$ to be separated and stored at $-20^{\circ} \mathrm{C}$ till it was used.

The ELISA procedure was followed according to the manufacturer's instructions. In brief, $50 \mu \mathrm{l}$ of the serum were incubated at $37^{\circ} \mathrm{C}$ for $60 \mathrm{~min}$ in 118 -well microplate coated with HBsAg reactive to HBsAb (anti-HBs). Subsequently, the wells were washed (three times) to remove residual serum. $50 \mu \mathrm{l}$ of anti-HBs conjugated $\mathrm{AB}$ was added and incubated at $37^{\circ} \mathrm{C}$ for $60 \mathrm{~min}$. The wells were washed (three times) to eliminate unbound conjugate, $50 \mu \mathrm{l}$ of enzyme-substrate and chromogen were added and incubated at $37^{\circ} \mathrm{C}$ for $60 \mathrm{~min} ; 50 \mu \mathrm{l}$ diluted stop solution (Sulphuric acid) was added and the plate was read at $450 \mathrm{~nm}$ as indicated by the manufacturer.

\section{Interpretation of the result}

The cut-off value was estimated by calculating the mean $(\mathrm{m})$ absorbance value of negative control $\times 2.1$.

Cut-off $=$ mean of the negative control $\times 2.1$

For the validity of the accomplishment, the following is required:

(a) Negative control means: absorbance of individual negative control values must be $<0.9$

(b) Positive control means: it must be $>1.1$

(c) Equivocal means: absorbance of individual equivocal values must be between 0.9 and 1.1. All statistical calculations were done using SPSS for Windows, version 24. 


\section{Results}

A total of 106 health personnel participated in this study, of those, 77 (72.6\%) were females and $29(27.4 \%)$ males with a female:male ratio of 2.65:1. The mean age of the participants was 27.69 years, the minimum age was 20 years, and the maximum age was 55 years.

Furthermore, 56 (52.8\%) participants were nurses by profession, 35 (33\%) were doctors, 4 (3.7\%) lab technicians, and 11 (10.4\%) others.

About 97 (91.5\%) participants took the last dose of vaccine within the past 10 years and $12(11.3 \%)$ took their vaccine during childhood.

The number of subjects who completed the all three doses of vaccine or more were $60(56.6 \%)$, those who took two doses were 23 (21.7\%), and those with only one dose were 23 (21.7\%), see Table 1.

TABLE 1: Total Number of HBV Vaccine Doses Received vs Immunity Acquired After Each Dose.

\begin{tabular}{|l|c|c|c|}
\hline $\begin{array}{l}\text { Number of HBV Vaccine } \\
\text { Doses Received }\end{array}$ & Frequency & Percent & Valid Percent \\
\hline $\mathbf{1}$ dose & $5.20+1.31$ & $4.28+0.88$ & 0.0076 \\
\hline $\mathbf{2}$ doses & $2.03+1.43$ & $1.26+0.76$ & 0.0271 \\
\hline $\mathbf{3}$ doses & $3.06+0.73$ & $2.60+0.56$ & 0.0248 \\
\hline $\mathbf{3}$ doses & $1.12+0.29$ & $1.20+0.24$ & 0.2736 \\
\hline Total & 106 & $100 \%$ & $100 \%$ \\
\hline
\end{tabular}

Sixty participants completed the three or more doses of vaccine, about forty two (66\%) had HBsAbs titer $\geq 10 \mathrm{mlU} / \mathrm{mL}$ and eighteen (30\%) had titer $<10 \mathrm{mlU} / \mathrm{mL}$. Of those who took two doses of the vaccine, 17 (73.9\%) had HBsAbs titer >10 mIU/L and 6 (26.1\%) $<10 \mathrm{mIU} / \mathrm{mL}$. And of those who received one dose, 12 (52.1\%) had titer $<10 \mathrm{mlU} / \mathrm{mL}$ and $11(47.8 \%) \geq 10 \mathrm{mIU} / \mathrm{mL}$ (Figure 1).

About 34 (32\%) participants were exposed to needle stick injury during their work but didn't received treatment, 3 (8.8\%) had undergone blood transfusion, and 1 had received Hepatitis B Immunoglobulin after accidental needle injury from Hepatitis B-infected patient and her HBsAb result was excellent at $191.0 \mathrm{mIU} / \mathrm{mL}$ (Figure 2).

\section{Discussion}

HCWs are at a higher risk of blood-borne infections, especially HBV. Vaccination is effective in protecting 90-95\% of adults [9]. According to the World Health Organization (WHO) estimate, hepatitis B vaccination coverage among healthcare workers varies from $18 \%$ being lowest in Africa to the highest $77 \%$ in Australia and New Zealand [10]. 


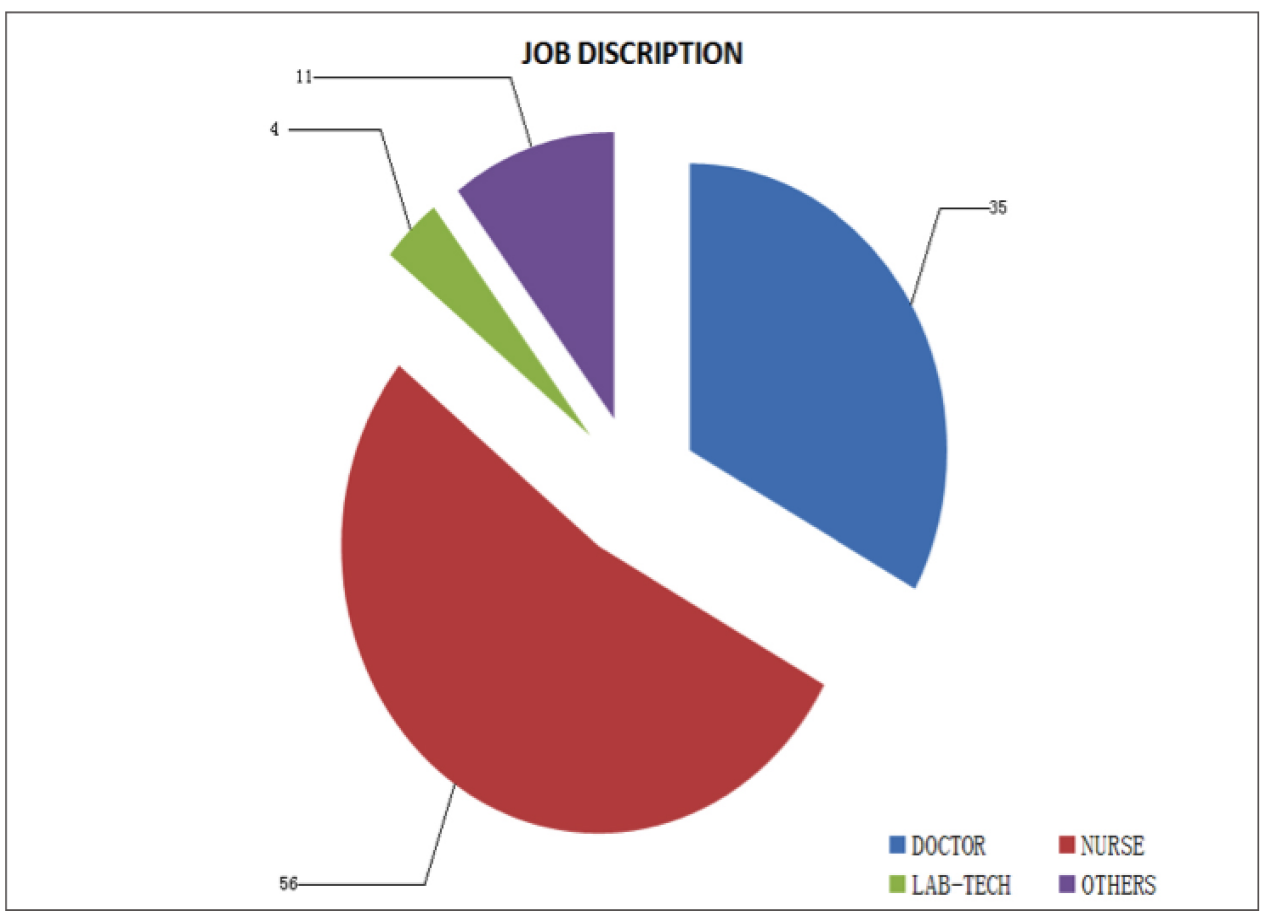

Figure 1: Distribution of Subjects Participating in this Study According to Specialty in Which Nurses (56) Were More than all the Other Groups.

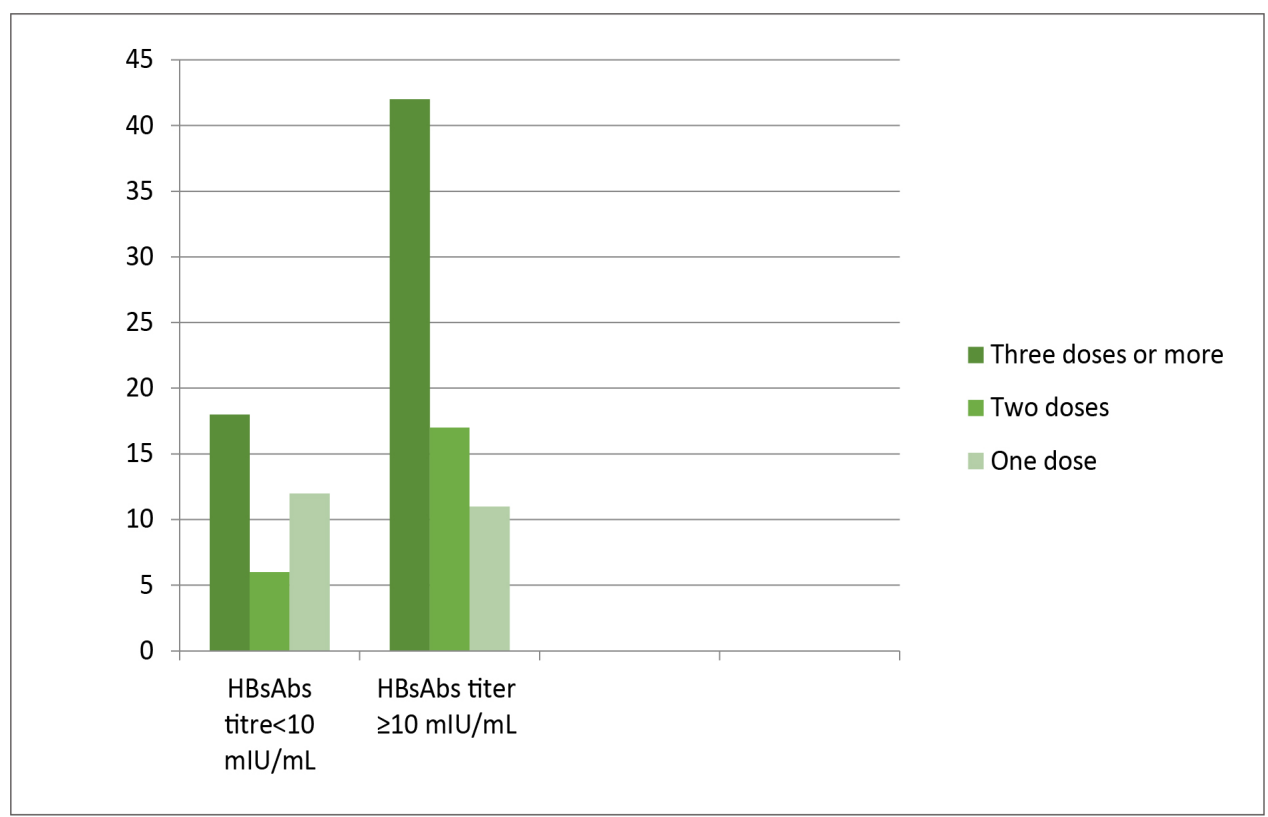

Figure 2: Correlation Between HBsAb Titer and Number of Participants Receiving Different Doses of HBV Vaccine $(p=0.035)$.

In this study, we examined the level of anti-HBsAb in healthcare personnel in three public hospitals in Khartoum state besides Khartoum doctors' mess; the hospitals were the Ibn Sina Specialized Hospital, the Alshaab Teaching Hospital, and the Academic Teaching Hospital. They were chosen mainly because of their easy accessibility. In this study, 60 (56.6\%) participants completed all three doses of vaccine, which is higher 
compared to the lower vaccination rates found in the other two studies conducted in Ethiopia, one in the Gondar University Hospital (28.7\%) [11] and the other at the Adama General Hospital and Medical College (25.6\%) [12] while nearly similar results as ours was seen in a study conducted in the Makueni County of Kenya (48\%) [13]. Another study reveals a higher percentage of full vaccination (72\%) in Libya [14].

From our results, we notice that there is a direct relationship between the numbers of doses the participants received and their immune status against HBV $(p=0.035)$, so those who received three or more doses of the vaccine, their results represented immunity by $70 \%$. On the other hand, just $47.8 \%$ of those who took one dose of HBV vaccine in their lifetime are immune to the virus (Table 1). In our study about $70 \%$ of the subjects were immuned after receiving at least three doses of the vaccine which is lower than the study done in West Pomeranian Region of Poland (94.5\%) [15]. Another finding in this study was that there is no big difference in HBV immunity between those who took their last dose of the vaccine $<10$ years ago (64.95\% are immune) and the participants who took their last vaccine dose $\geq 10$ years ago ( $77.8 \%$ are immune). In general, about $47.2 \%$ (50) of the participants in this study did show excellent immune response (>100 mlU/mL), 18.9\% (20) had weak protective levels (between 10 and $100 \mathrm{mlU} / \mathrm{mL}$ ) which in comparison to the Lasemi, Eshagh et al's study, were a little bit higher as in their study, 54\% of the students had an excellent protective immune response and $24.2 \%$ had a positive but weak immune response [16]. Some suggest that the weak immune response among health personnel in Sudan may be due to the type of vaccine that is used, which is an imported vaccine from outside the country. No official data about the type of vaccine are available from the National Medical Supplies Fund (NMSF). Only 56.6\% of the involved health personnel finished the recommended three doses that reflects the gap in the health education and awareness about HBV vaccination among health personnel. This may be due to the high cost and lack of a public protocol for health personnel HBV vaccination, we found only two previous researches close to this topic in Sudan [17]. It is worth mentioning that most participants 56 (52.8\%) were nurses, who were more willing to get involved and check their HBsAb levels than doctors.

About 70 (66.0\%) participants were surveyed at the Ibn Sina Hospital; they were eager to get involved because they wanted to know their immune status against HBV as the hospital is the main referral hospital for gastroenterology and hepatology in Sudan. They have better awareness about the importance of vaccination against the HBV and their increased risk of acquiring the disease if they don't have immunity against the virus especially with the high numbers of hepatitis B patients in their hospital. 


\section{Conclusion}

From this research, we can conclude that HBV post-vaccination immunity status of health personnel in Khartoum state is lower than the post-vaccination HBV immunity in other parts of the world. So, there is an urgent need for a national protocol of HBV vaccination to improve the immunization status of healthcare workers that includes regular checkups of HBV immunity and easy access to a good-quality vaccine.

To get more accurate results of the response to the hepatitis $B$ vaccine among healthcare workers in Sudan, more large-scale studies should be conducted.

\section{References}

[1] Liang, TJ (2009). Hepatitis B: the virus and disease. Hepatology. 49(5): S13-S21.

[2] Ganem D, Prince AM (2004). Hepatitis B virus infection-natural history and clinical consequences. N Engl J Med. 350(11): 1118-1129.

[3] WHO (2016). Hepatitis B. Fact Sheet.

[4] Mudawi HM (2008). Epidemiology of viral hepatitis in Sudan. Clin Exp Gastroenterol. 1: 9-13.

[5] Hamadalnil Y, Bakheit S (2017). Hepatitis B virus_surface gene mutations and their clinical implications. Sudan J Med Sci. 12(2): 101-13.

[6] Nelson NP, Easterbrook PJ, McMahon BJ (2016). Epidemiology of hepatitis B virus infection and impact of vaccination on disease. Clin Liver Dis. 20(4): 607-628.

[7] Bagheri-Jamebozorgi M, Keshavarz J, Nemati M, Mohammadi-Hossainabad S, Rezayati MT, Nejad-Ghaderi M et al. (2014). The persistence of anti-HBs antibody and anamnestic response 20 years after primary vaccination with recombinant hepatitis B vaccine at infancy. Hum Vaccin Immunother. 10(12): 3731-3736.

[8] Yang S, Tian G, Cui Y et al. (2016). Factors influencing immunologic response to hepatitis B vaccine in adults. Sci Rep. 6: 27251.

[9] Coppola N, Onorato L, Minichini C et al. (2015). Clinical significance of hepatitis B surface antigen mutants. World J Hepatol. 7(27): 2729-2739.

[10] Pruss-Ustun A, Rapiti E, Hutin Y (2005). Estimation of the global burden of disease attributable to contaminated sharps injuries among health-care workers. The Am J Ind Med. 48(6): 482-490.

[11] Ayalew MB, Horsa BA (2017). Hepatitis B vaccination status among health care workers in a tertiary hospital in Ethiopia. Hepat Res Treat. 2017: 6470658. 
[12] Akibu M, Nurgi S, Tadese $M$ et al. (2018). Attitude and vaccination status of healthcare workers against hepatitis B infection in a teaching hospital, Ethiopia. Scientifica. 2018: 6705305.

[13] Kisangau EN, Awour A, Juma B et al. (2018). Prevalence of hepatitis B virus infection and uptake of hepatitis B vaccine among healthcare workers. J Public Health (Oxf). 41(4): 765-771.

[14] Ziglam H, El-Hattab M, Shingheer N et al. (2013). Hepatitis B vaccination status among healthcare workers in a tertiary care hospital in Tripoli, Libya. $J$ Infect Public Health. 6(4): 246-251.

[15] Ganczak M (2012). A cross-sectional study on anti-hepatitis B immune status in vaccinated healthcare workers in the West Pomeranian Region of Poland. Hepat Mon. 12(3): 185-189.

[16] Lasemi E, Haddadpour N, Navi F et al. (2011). Rate of acquired immunity in dental students after hepatitis B vaccination. Dent Res J. 8(3): 128-131.

[17] Abdel A, Khamis G, Jacoub AO et al. (2014). Knowledge, attitudes and practices of surgical staff at Khartoum State hospitals towards Hepatitis B infection. Khartoum Med J. 7(1): 932-940. 